\title{
A utilização da dermatoscopia na Medicina Veterinária: Uma revisão sistemática de
}

\section{literatura}

\author{
The use of dermatoscopy in Veterinary Medicine: A systematic literature review \\ El uso de la dermatoscopía en la Medicina Veterinaria: Revisión sistemática de la literatura
}

Giselle Almeida Couceiro

ORCID: https://orcid.org/0000-0003-1878-669X Universidade Federal Rural da Amazônia, Brasil E-mail: gisellecouceiro@gmail.com

Sinerey Karla Salim Aragão de Sousa ORCID: https://orcid.org/0000-0002-3994-9358 Universidade Federal Rural da Amazônia, Brasil E-mail: sinerey@globo.com

Leandro Nassar Coutinho

ORCID: https://orcid.org/0000-0002-9579-3052 Universidade Federal Rural da Amazônia, Brasil E-mail: leandro.nassar@ufra.edu.br

\begin{abstract}
Resumo
A dermatoscopia é um método diagnóstico realizado in vivo, de caráter não invasivo, rápida execução, indolor e não agride a pele. É utilizada como exame complementar e permite a observação de estruturas da epiderme, da junção dermoepidérmica e da derme papilar e reticular superficial, sendo considerada um elo entre o quadro clínico (macroscópico) e o exame histopatológico. Com o objetivo de realizar uma revisão sistemática dos artigos publicados com dermatoscopia em animais, através de uma revisão de literatura dos conceitos, técnicas e aplicações deste exame na Medicina Veterinária, foi realizada uma pesquisa eletrônica utilizando as bases de dados PubMed, Scielo, CAFe, Wiley online library e Google scholar. Foram encontrados 12 artigos, $5(41,66 \%)$ sobre caninos, $4(33,33 \%)$ sobre felinos e $3(25 \%)$ sobre equinos. Estes trabalhos utilizaram a dermatoscopia, na avaliação da pele felina e equina normal, dermatofitose felina e canina, padrão de alopecia em cães, malformações linfáticas dérmicas superficiais congênitas acrais em gatos e descrição de padrão colorimétrico em cães com eritroderma. Através desta pesquisa, demonstrou-se que a dermatoscopia ainda é incipiente na Medicina Veterinária, porém com resultados promissores. $\mathrm{O}$ exame dermatoscópico revelou-se útil para descrever a pele normal, como triagem e auxiliar à conclusão do diagnóstico de algumas doenças de pele em animais. Embora observe-se o interesse de pesquisadores pelo assunto, e a potencialidade do exame, ainda se faz necessária a realização de mais pesquisas que identifiquem novas aplicabilidades da dermatoscopia e padrões dermatoscópicos dentre as diversas dermatopatias que acometem animais. Palavras-chave: Dermoscopia; Dermatologia; Dermatopatias; Exame.
\end{abstract}

\begin{abstract}
Dermoscopy is a non-invasive diagnostic method performed in vivo, is quick, painless and does not harm the skin. It is used as a complementary exam and allows the observation of structures of the epidermis, the dermoepidermal junction and the superficial papillary and reticular dermis, being considered a link between the clinical picture (macroscopic) and the histopathological exam. In order to conduct a systematic review of articles published with dermoscopy in animals, through a literature review of the concepts, techniques and applications of this examination in Veterinary Medicine, an electronic search was carried out using the databases PubMed, Scielo, CAFe, Wiley online library and Google scholar. Twelve articles were found, $5(41,66 \%)$ about canines, $4(33,33 \%)$ about felines and 3 (25\%) about horses. These studies used dermoscopy in the evaluation of normal feline and equine skin, feline and canine dermatophytosis, pattern of alopecia in dogs, acral congenital superficial dermal lymphatic malformations in cats and description of colorimetric pattern in dogs with erythroderma. Through this research, it was demonstrated that Dermatoscopy is still incipient in Veterinary Medicine, but with promising results. The dermoscopic examination proved to be useful to describe normal skin, as a screening and to aid in the conclusion of the diagnosis of some skin diseases in animals. Although researchers are interested in the subject, and the exam's potential, it is still necessary to carry out more research to identify new applicability of dermoscopy and dermoscopic patterns among the different skin diseases that affect animals.
\end{abstract}

Keywords: Dermoscopy; Dermatology; Dermathopathies; Exam. 


\begin{abstract}
Resumen
La dermatoscopia es un método de diagnóstico no invasivo, realizado in vivo, rápido, indoloro y no daña la piel. Se utiliza como examen complementario y permite la observación de estructuras de la epidermis, unión dermoepidérmica y dermis papilar y reticular superficial, considerándose un vínculo entre el cuadro clínico (macroscópico) y el examen histopatológico. Para realizar una revisión sistemática de los artículos publicados con dermatoscopia en animales, mediante de una revisión bibliográfica de los conceptos, técnicas y aplicaciones de este examen en Medicina Veterinaria, se realizó una búsqueda electrónica utilizando las bases de datos PubMed, Scielo, CAFe, Wiley online library y Google scholar. Se encontraron 12 artículos, 5 (41,66\%) sobre caninos, $4(33,33 \%)$ sobre felinos y 3 (25\%) sobre caballos. Estos estudios utilizaron dermatoscopia en la evaluación de piel felina y equina normal, dermatofitosis felina y canina, patrón de alopecia en perros, malformaciones linfáticas dérmicas superficiales congénitas acrales en gatos y descripción del patrón colorimétrico en perros con eritrodermia. Mediante esta investigación se demostró que la dermatoscopia aún es incipiente en Medicina Veterinaria, pero con resultados prometedores. El examen dermatoscópico demostró ser útil para describir la piel normal, como cribado y para ayudar a la conclusión del diagnóstico de algunas enfermedades de la piel en animales. Aunque los investigadores están interesados en el tema y el potencial del examen, aún es necesario realizar más investigaciones para identificar una nueva aplicabilidad de la dermatoscopia y los patrones dermatoscópicos entre las diferentes enfermedades de la piel que afectan a los animales.

Palabras clave: Dermoscopia; Dermatología; Dermatopatías; Examen.
\end{abstract}

\title{
1. Introdução
}

As dermatopatias constituem as enfermidades mais frequentes na clínica de pequenos animais e o exame clínico, na maior parte dos casos, não é suficiente para estabelecer um diagnóstico (Wilkinson, Harvey, 1996, Mueller, 2003). Os métodos diagnósticos de afecções da pele variam da observação clínica dos sintomas a exames laboratoriais (Barboza et al. 2019).

A dermatoscopia é um exame que tem uso crescente em todas as áreas, principalmente na Medicina Humana (Menzies, 2013). É um método diagnóstico realizado in vivo, de caráter não invasivo, de rápida execução, indolor e que não agride a pele dos animais. É utilizada como exame complementar e permite a observação de estruturas da epiderme, da junção dermoepidérmica e da derme papilar e reticular superficial (Malvehy et al., 2007, Scarampella et al., 2015).

Estudos dermatoscópicos foram realizados em animais com lesões de pele, como alopecia congênita ou adquirida, dermatofitose, malformação linfática e eritema, e desde então, têm-se ampliado o interesse acerca deste tema. Nos últimos anos, alguns estudos mostraram que a dermatoscopia pode ser útil para auxiliar o diagnóstico de distúrbios dermatológicos, acompanhamento e monitoramento terapêutico (Lallas et al., 2012, Errichetti et al, 2015, Lacarruba et al, 2016). Diante disto, o objetivo deste estudo foi realizar uma revisão sistemática dos artigos publicados com dermatoscopia em animais, através de uma revisão de literatura dos conceitos, técnicas e aplicações deste exame na Medicina Veterinária.

\section{Revisão de Literatura}

\subsection{Conceito}

A dermatoscopia digital, também conhecida como teledermatoscopia ou videodermatoscopia é o exame dermatológico realizado com o emprego de uma videocâmera que capta as imagens e transfere via linguagem binária para o computador, vídeo ou tela LCD acoplada e para utilização em smartphones (Paschoal, 2000, Castells, 2014) que mantém a definição da imagem e possibilita armazenar as imagens digitais (Rezze, Sá, Neves, 2006) permitindo o acompanhamento e comparação de lesões ao longo do tempo. Este método está ganhando cada vez mais popularidade, porque pode ser amplamente aplicado em diagnóstico diferencial de doenças do cabelo e do couro cabeludo (tricodermatoscopia) (Rudnicka, Rakowska, Olszewska, 2013). Etimologicamente, o termo dermo significa pele e scopia vem do grego scopein que significa estudo (Avilés, 2010). A Dermatoscopia, também chamada de dermoscopia, microscopia de epiluminescência, microscopia de luz reflexa ou microscopia de superfície da pele é uma técnica não invasiva, executada in vivo (Argenziano et al., 2004), facilmente aplicável na prática clínica e de custo relativamente baixo (Soyer et al., 2001). 


\subsection{Técnica}

A imagenologia médica transformou drasticamente a maneira como os médicos avaliam, diagnosticam, monitoram e tratam doenças. A natureza altamente visual das doenças cutâneas torna a imagem digital valiosa na prática cotidiana da dermatologia (Tkaczyk, 2017).

A dermatoscopia é utilizada como exame complementar à avaliação clínica e permite a observação de estruturas da epiderme, da junção dermoepidérmica e da derme papilar e reticular superficial, não observáveis a olho nu (Malvehy et al., 2007, Scarampella et al., 2015), por esta razão é geralmente considerada um elo entre o quadro clínico (macroscópico) e exame histopatológico (Braun et al., 2005, Cutolo, Pizzorni, Sulli, 2005).

A prática da dermatoscopia tradicional se baseava no uso do clássico dermatoscópio com luz não-polarizada, associado à interface de imersão líquida em contato direto com a pele. Os dermatoscópios originais consistiam em uma peça ocular de magnificação (x 10) com uma fonte de luz conectada ao cabo de um otoscópio que atingia a superfície cutânea em um ângulo de $20^{\circ}$ e permitia um aumento variável de seis a 400x (Rezze, Sá, Neves, 2004). Existem também aparelhos dermatoscópicos que não necessitam de interface de imersão, utilizam luz polarizada e permitem uma melhor visualização da vascularização (Benvenuto et al., 2007, Arrazola et al. 2015). Existem três modalidades de execução do exame: 1. dermatoscopia não polarizada, 2. dermatoscopia polarizada de contato e 3. dermatoscopia polarizada sem contato. A dermatoscopia não polarizada exige contato entre o escopo e a pele, além de uma interface líquida colocada entre o dermoscópio e a pele, a dermatoscopia polarizada pode ser usada com ou sem contato e usa filtros de polarização cruzada, o que elimina a luz refletida da superfície da pele (Pan Y et al., 2008).

Entre a superfície da pele e a lâmina de vidro do aparelho pode-se aplicar um fluido (óleo, água, gel, álcool-gel ou glicerina) para eliminar a reflexão normal da luz no extrato córneo e melhorar a translucência da epiderme (Braun et al., 2005, Hischal \& Khopkar, 2005). Isso permite a observação das características dermatoscópicas resultantes da melanina e hemoglobina nos diferentes níveis da epiderme e derme (Carmo, Silva, 2008). A luz direcionada para a pele é refletida, dispersa ou absorvida pelo estrato córneo devido às suas características de refração e sua densidade óptica que são diferentes do ar (Braun, French, Saurat, 2004, Hanke, 2012).

A lente de vidro deve ser cuidadosamente colocada sobre a lesão e uma pressão mínima deve ser aplicada ao usar dermoscópios de contato. No entanto, é melhor evitar tais aplicações em dermoscópios de contato, uma vez que esses dispositivos ópticos podem exigir a aplicação de sobre pressão na lesão para obtenção de imagem (Zalaudek et al, 2010).

Em Medicina Veterinária, devido à abundância de pelos dos animais, devem-se buscar zonas glabras ou tentar afastar os pelos para avaliar lesões, que devem estar secas e o ponto de aplicação do dermatoscópio terá que ser o mais plano possível (Goth, 2011).

Obviamente, achados dermatoscópicos devem ser interpretados à luz do quadro clínico geral e contexto do paciente (história pessoal / familiar, sintomatologia, quantidade, localização, morfologia e distribuição de lesões, etc.) porque apenas a combinação entre esses dados pode realmente melhorar a precisão do diagnóstico no campo de distúrbios dermatológicos gerais (Zalaudek et al, 2006, Lallas et al., 2013b, Sgouros et al, 2018).

\subsection{Aplicações gerais na Medicina Humana}

A dermatoscopia tem como objetivo principal definir critérios para lesões pigmentadas (Nachbar et al., 1994) e auxiliar o diagnóstico de lesões cutâneas (melanocíticas ou não) (Rezze, Sá, Neves, 2006). Também é utilizada para a avaliação de padrões vasculares, pelos e anormalidades foliculares (Zalaudek et al, 2006, Rudnicka et al, 2012, Miteva \& Tosti, 2012), nas doenças infecciosas e inflamatórias da pele (inflamoscopia) (Lallas et al., 2012), alopecia cicatricial, urticária, 
vasculite, doenças do colágeno, entodermatoscopia (Malvehy et al., 2007), desordens escamosas, despigmentações,"pseudofoliculite" (Zalaudek et al., 2008) e dermatite seborréica (Leite Júnior, 2012).

Pode também ser útil para triagem de familiares, monitorização da resposta a diversos tratamentos, acompanhamento pós-terapêutico (Lallas et al, 2013a, Micali et al, 2018b) e pode auxiliar no estabelecimento do diagnóstico já sendo utilizada para avaliar a resposta ao tratamento em várias dermatopatias humanas (Lacarruba et al., 2010). Suas aplicações clínicas vêm sendo expandidas constantemente e incluem: triagem, diagnóstico, avaliação prognóstica e monitoramento do tratamento de vários anexos cutâneos e desordens cutâneas (Micali et al., 2018a).

\section{Metodologia}

O método utilizado para o levantamento dos dados baseou-se em aspectos da Revisão Sistemática de Literatura, que é um método de pesquisa que se propõe a investigar um determinado tema, a partir de uma busca literária sistematizada, identificar sistematicamente todos os documentos relevantes para uma questão de investigação, avalia a qualidade e quantidade desses artigos, extrai os dados e sintetiza os resultados, sendo de grande valia para a orientação de demais investigações (Donato, \& Donato, 2019; Siddaway et al. 2019).

Foi realizada uma pesquisa eletrônica utilizando as bases de dados PubMed, Scielo, CAFe, Wiley online library e Google scholar, utilizando como termo de procura as palavras ["dermoscopy, dermoscopic, dermatoscopy, dermatoscopic, dermoscopia, dermatoscopia"] com termos adicionais [“dog, cat, horse, canine, feline, equine”]. Os critérios de inclusão foram artigos originais, independente da data de publicação e idioma.

\section{Resultados}

Foram encontrados 12 artigos, $5(41,66 \%)$ sobre caninos, $4(33,33 \%)$ sobre felinos e $3(25 \%)$ sobre equinos. Estes trabalhos foram selecionados e revisados, sendo possível ter acesso na área de Medicina Veterinária, a trabalhos sobre dermatoscopia, com uso na avaliação da pele felina normal, dermatofitose felina e canina, padrão de alopecia em cães (Scarampella et al., 2015, Dong et al., 2016, Zanna et al., 2015, Zanna et al., 2016, Scarampella et al., 2017, Scarampella \& Roccabianca, 2018), avaliação de pele equina normal (Legnani et al., 2017, Tomich et al., 2017, Zniszczynska et al., 2021), malformações linfáticas dérmicas superficiais congênitas acrais em gatos (Legnani et al. 2020) e descrição de padrão colorimétrico em cães com eritroderma (Cugmas \& Struc, 2020, Cugmas et al., 2021).

\section{Discussão}

Nesta revisão pôde-se observar que os estudos com dermatoscopia na Medicina Veterinária ainda são incipientes em comparação com a Medicina Humana.

Na pele equina normal, o exame dermatoscópico foi descrito como viável e de fácil execução e foram observados fios de pelos lisos e espessos emergindo de aberturas foliculares únicas. Fios da cabeça e crina eram significativamente mais espessos, mas menos densos em comparação com outras regiões, pigmentação da pele e descoloração perifolicular variáveis também foram observadas (Legnani et al., 2017). A dermatoscopia de luz polarizada permitiu a visualização de poros deprimidos distintos das aberturas foliculares em 10 de 12 cavalos avaliados. O exame histológico demonstrou que esses poros se correlacionavam com aberturas ductulares das glândulas sudoríparas, uma descoberta raramente relatada em cavalos. A maioria dos indivíduos tinha pele com pigmentação escura com ocasionais áreas de pele não pigmentada e na pele não pigmentada, fios de pelo dentro da porção infundibular do folículo capilar eram visíveis sob a superfície da pele (Tomich et al., 2017). 
Zniszczynska et al. (2021) utilizaram a videodermatoscopia para avaliar a pele e pelos de éguas puras árabes durante o inverno, revelando diferenças pigmentares, com vasos sanguíneos visíveis na pele não pigmentada, bem como diferentes densidades e espessuras dos pelos em áreas corporais distintas.

Avaliando a pele felina normal, com um videodermoscópio com ampliação de 70 vezes, Zanna et al. (2015) observaram pelos primários grossos e lisos cercados por múltiplos pelos secundários. A espessura da haste do pelo foi medida e as aberturas foliculares e disposição de vasos arborizantes foram claramente observados. No ventre os pelos mais finos eram mais numerosos e os pelos primários do dorso, mais grossos e finos emergiam individualmente de óstios foliculares únicos. O exame permitiu a visualização do infundíbulo do folículo piloso e da pele interfolicular.

Pelos parecidos com vírgulas e saca-rolhas, opacos, ligeiramente curvados e quebrados caracterizados por uma espessura homogênea foram descritos como as principais características dermatoscópicas da dermatofitose felina devido a Microsporum canis e Microsporum gypseum (Scarampella et al., 2015, Dong et al., 2016) demonstrando que mesmo que a cultura fúngica continue sendo o padrão-ouro para diagnóstico de dermatofitose, tanto em humanos como em animais, a dermatoscopia pode auxiliar na avaliação clínica inicial (Pinheiro et al., 2012). Já em cães com dermatofitose, todos apresentaram alopecia multifocal e variável quantidade de escamas. Os achados mais comuns observados na dermatoscopia eram pelos opacos e quebrados, caracterizados por uma afilação e inclinação na porção final, espessura homogênea (cinco de seis cães), e escamas brancas / amarelas grandes (Scarampella et al., 2017).

Scarampella et al. (2015) descreveram que algumas das características dermatoscópicas observadas em gatos com alopecia autoinduzida foram semelhantes àqueles relatados em pessoas com tricotilomania, pelos quebrados com comprimentos diferentes foram comumente vistos.

O padrão de alopecia canina foi avaliado por dermatoscopia em 20 cães de pelos curtos além da descrição de características dermatoscópicas de um caso clínico de cão com alopecia areata com afinamento da haste do pelo, pelos circulares e tampões de queratina folicular, nas áreas expostas ao sol afetadas, havia também um padrão de pigmentação semelhante a um favo de mel (Zanna et al., 2016). Na alopecia areata foram observados obstrução do infundíbulo folicular com um material amarelo-marrom, poucos pelos quebrados, e pelos curtos que voltam a crescer surgindo individualmente dos óstios foliculares, rede pigmentada semelhante a favo de mel também foi detectada na pele interfolicular exposta ao sol (Scarampella \& Roccabianca, 2018).

Legnani et al. (2020) descreveram achados clínico-patológicos e dermatoscópicos de malformações linfáticas dérmicas superficiais congênitas acrais em dois gatos. Observaram lacunas translúcidas a avermelhadas demarcadas por bordas mais claras e transição de cor de vermelho escuro para amarelado e branco visível (sinal do tipo hipópio), estruturas alongadas de rosa a vermelho escuro a serpiginosas (lacunas e espaços vasculares).

Cugmas e Struc (2020), avaliaram a utilização da teledermatoscopia através de smartphone para medição de cores com padrão colorimétrico da pele canina nativa e eritematosa, demonstrando que a pele canina é menos avermelhada e amarelada que a pele humana padrão caucasiana. Concluindo que o sistema de teledermoscopia calibrado é rápido e preciso o suficiente para várias aplicações colorimétricas em dermatologia veterinária. Ainda na área de colorimetria, foi avaliada a pele inguinal eritematosa não pigmentada de cães, com diagnóstico de dermatite atópica, estudo de Cugmas et al. (2021) indicou que a dermatoscopia baseada em smartphone pode ser uma maneira conveniente e precisa de avaliar a gravidade do eritema cutâneo.

\section{Considerações Finais}

A Dermatoscopia ainda é incipiente na Medicina Veterinária, porém com resultados promissores e de relevante potencialidade. 
Com a importância da utilização de métodos cada vez mais práticos, rápidos, seguros e menos invasivos, a dermatoscopia pode representar uma valiosa técnica para o exame dermatológico do paciente veterinário. O exame dermatoscópico revelou-se útil para descrever a pele normal, como triagem e auxiliar à conclusão do diagnóstico de algumas doenças de pele em animais, mesmo considerando que para a maioria das lesões descritas, não existem ainda critérios dermatoscópicos padronizados na Medicina Veterinária. Ressalta-se que a característica dermatoscópica de qualquer dermatopatia deve ser avaliada levando em consideração o contexto clínico geral da mesma.

Embora observe-se o interesse de pesquisadores pelo assunto, e a potencialidade do exame, ainda se faz necessária a realização de mais pesquisas que identifiquem novas aplicabilidades da dermatoscopia e padrões dermatoscópicos dentre as diversas dermatopatias que acometem animais.

\section{Referências}

Argenziano, G., Zalaudek, I., Corona, R., Sera, F., Cicale, L., Petrillo, G., Ruoco, E., Hofmann-Wellenhof, R. \& Soyer, H. P. (2004). Vascular structures in skin tumors: a dermoscopy study. Archives of Dermatology, 140, 1485-1489.

Arrazola, G. J., Isa, I. R., Torres, G. E. \& Arenas, R. (2015). Tinea capitis. Dermoscopic findings in 37 patients. Revista Iberoamericana de Micología. $32: 242-6$.

Avilés, D. (2010). Impacto del desarollo socioeconómico en la resolución de investigaciones forenses en Contribuciones a las Ciencias Sociales. http://www.eumed.net/rev/cccss/07/daa6.htm

Barboza, W. G. A., Souza, E. W. \& Paulino, A. M. (2019). Dermatite atópica em uma cadela: caso clínico. PUBVET. 13, a457, 1-7.

Benvenuto, A. C., Duzza, S. W., Agero, A. L., Scope, A., Rajadhyaksha, M., Halpern, A. C. \& Marghoob, A. (2007). A. Differences between polarized light dermoscopy and immersion contact dermoscopy for the evaluation of skin lesions. Archives of Dermatology, 143, 329-38.

Braun, R.P., French, L.E. \& Saurat, J.H. (2004). Dermoscopy of pigmented lesions: a valuable tool in the diagnosis of melanoma. Swiss Medical weekly, 134, 83-90.

Braun, R. P., Rabinovitz, H. S., Oliviero, M., Kopf, A. W. \& Saurat, J. H. (2005). Dermoscopy of pigmented skin lesions. Journal of the American Academy of Dermatology. 52:109-121.

Carmo, G.C. \& Silva, M.R. (2008). Dermoscopy: basic concepts. International Journal of Dermatology, 47(7), 712-9.

Castells. (2014). Desenvolvimento óptico. http://www.castells.com.br/quemsomos

Cutolo, M., Pizzorni, C. \& Sulli, A. (2005). Capillaroscopy. Best practice \& Research clinical rheumatology, 19 (3), $437-452$.

Cugmas, B., Viskere, D., Struc, E. \& Olivry, T. (2021). Evaluation of erythema severity in dermatoscopic images of canine skin: Erythema index assessment and image sampling reliability. Sensors, 21(4), 1285.

Cugmas, B. \& Struc, E. (2020). Accuracy of an affordable smartphone-based teledermoscopy system for color measurements in canine skin. Sensors, 20, 6234 .

Donato,H.. \& Donato, M. (2019). Etapas na Condução de um Revisão Sistemática. Revista Acta Medica Portuguesa, 32(3): 227-35.

Dong, C., Angus, J., Scarampella, F. \& Neradilek, M. (2016). Evaluation of dermoscopy in the diagnosis of naturally occurring dermatophytosis in cats. Veterinary Dermatology. 27: 275-e65.

Errichetti, E., Lacarrubba, F., Micali, G. et al. (2015). Differentiation of pityriasis lichenoides chronica from guttate psoriasis by dermoscopy. Clinical and Experimental Dermatology. 40 (7):804-6.

Goth, G. M. (2011). Dermatología canina y felina. Manuales clínicos por especialidades. Editorial Servet. Navarra: Espanha, 328p.

Hanke, M. (2012). Introdução à Dermatoscopia. http://pt.scribd.com/doc/98086938/Introducao-a-Dermatoscopia-aula16-02-12parte1

Hischal, K.C.\& Khopkar, U. (2005). Dermoscope. Focus, 71(4), 300-3.

Lacarrubba, F., D’amico, V., Nasca M. R. et al. (2010). Use of dermatoscopy and videodermatoscopy in therapeutic follow-up: a review. International Journal of Dermatology. 49(8): 866-73.

Lacarrubba, F., Musumeci, M. L., Ferraro, S. et al. (2016). A three-cohort comparison with videodermatoscopic evidence of the distinct homogeneous bushy capillary microvascular pattern in psoriasis vs atopic dermatitis and contact dermatitis. Journal of the European Academy of Dermatology and Venereology. 30 (4):701-3.

Lallas, A. et al. (2013a). Dermoscopy of early-stage mycosis fungoides. Journal of the European Academy of Dermatology and Venereology. $27,617-21$. 
Lallas, A., Kyrgidis, A., TzelloS, T. G., Apalla, Z., Karakyriou, E., Karatolias, A., Lefaki, I., Sotiriou, E., Ioannides, D., Argenziano, G. \& Zalaudek, I. (2012). Accuracy of dermoscopic criteria for the diagnosis of psoriasis, dermatitis, lichen planus and pityriasis rosea. British Journal of Dermatology, v. 166 (6), 1198205 .

Lallas, A., Zalaudek, I., Argenziano, G., Longo, C., Moscarella, E., Di Lernia, V., Al Jabout, S. \& Apalla, Z. (2013b). Dermoscopy in general dermatology. Dermatologic Clinics. 31: 679-694.

Legnani, s., Abramo, f., Zanna, g., Graziano, L., Cornegliani \& L., Roccabianca, P. (2020). Acral congenital superficial dermal lymphatic malformations in two unrelated cats: Clinicopathological, Dermoscopic and ultrastructural findings. Veterinary Dermatology. 31 (4): 309 -e 77.

Legnani, s., Zini, E., Roccabianca, P., Funiciello, B. \& Zanna, G. (2017). Dermoscopic analysis of the skin of healthy warmblood horses: a descriptive study of 34 cases in Italy. Veterinary Dermatology. 29 (2): 165 -e61.

Leite Junior, A. C. (2012) Videodermoscopia Digital em Tricologia. http://tricologiamedica.blogspot.com.br/2012/04/curso-de-videodermatoscopia-em.html

Malvehy, J., Puig, S., Argenziano, G., Marghoob, A.A. \& Soyer, H. P. (2007). Dermoscopy report: proposal for standardization. Results of a consensus meeting of the International Dermoscopy Society. Journal of American Academy of Dermatology, v. 57(1), 84-95.

Menzies, S.W. (2013). Evidence-based dermoscopy. Dermatologic Clinics. 31: 521-524.

Miteva, M. \& Tosti, A. (2012). Dermoscopy guided scalp biopsy in cicatricial alopecia. Journal of the European Academy of Dermatology and Venereology, [in press].

Micali, G., Verzì, A. E. \& Lacarrubba, F. (2018a). Alternative uses of dermoscopy in daily clinical practice: An update. Journal of American Academy of Dermatology. v. 79 (6), 1117-1132.

Micali, G., Verzì, A. E., Quattrocchi, E., Chau Yee, N.G. \& Lacarrubba, F. (2018b). Dermatoscopy of Common Lesions in Pediatric Dermatology. Dermatologic Clinics. 36, 463-472.

Mueller, R. S. (2003). Dermatologia para o Clínico de pequenos animais. São Paulo: Roca, 162p, 2003.

Nachbar, F., Stoltz, W., Merkle, T., Cognetta, A. B., Vogt, T., Landthaler, M., et al. (1994). The ABCD rule of dermatoscopy: high prospective value in the diagnosis of doubtful melanocytic skin lesions. Journal of American Academy of Dermatology, v. 30, 551-9.

Pan, Y., Gareau, D. S., Scope, A., Rajadhyaksha, M., Mullani, N. A. \& Marghoob, A. (2008). Polarized and nonpolarized dermoscopy: the explanation for the observed differences. Archives of Dermatology. 144 (6): 828-9.

Paschoal, F.M. (2000). Dermatoscopia Digital. Boletim Informativo do Grupo Brasileiro de Melanoma. Ano III, 10.

Pinheiro, A. M. C., Lobato, L. A. \& Varella, T. C. N. (2012). Dermoscopy findings in tinea capitis. Case report and literature review. Anais Brasileiros de Dermatologia, v.87(2).

Rezze, G. G., Sá, B. C. S. \& Neves, R. I. (2004). Atlas de Dermatoscopia Aplicada. São Paulo: Lemar, p.19-109.

Rezze, G. G., Sá, B. C. S. \& Neves, R. I. (2006). Dermatoscopia: método de análise de padrões. Anais Brasileiros de Dermatologia, 3 , 261-8.

RudnickA, L., Olszewska, M. \& Rakowska, A. (2012). Trichoscopic structures and patterns. In: Rudnicka L, Olszewska M, Rakowska A, eds. Atlas of trichoscopy: dermoscopy in hair and scalp disease. London: Springer, 361-369.

Rudnicka, L., Rakowska, A. \& Olszewska, M. (2013). Trichoscopy How It May Help the Clinician. Dermatologic Clinics. $3129-41$.

Scarampella, F. \& Roccabianca, P. (2018). Alopecia Areata in a Dog: Clinical, Dermoscopic and Histological Features. Skin Appendage Disorders. 4: 112117.

Scarampella, F., Zanna, G., Peano, A. et al. (2015). Dermoscopic features in 12 cats with dermatophytosis and in 12 cats with self-induced alopecia due to other causes: an observational descriptive study. Veterinary Dermatology. 26: 282-e63.

Scarampella, F., Zanna, G. \& Peano, A. (2017). Dermoscopic features in canine dermatophytosis: some preliminar observations. Veterinary Dermatology. 28: $255-257$.

Sgouros, D., Apalla, Z., Loannides, D., Katoulis, A., Rigopoulos, D., Sotiriou, E., Stratigos, A., Vakirlis, E. \& Lallas, A. (2018). Dermoscopy of common inflammatory disorders. Dermatologic Clinics. 36, 359-368.

Siddaway, A. P, Wood, A. M. \& Hedges, L. V. (2019). How to do a systematic review: a best practice guide for conducting and reporting narrative reviews, meta-analyses, and meta-syntheses. Annual Review of Psychology. 70:747- 70.

Soyer, H. P., Argenziano, G., Talamini, R. \& Chimenti, S. (2001). Is dermoscopy useful for the diagnosis of melanoma? Archives of Dermatology, v. 137(10), p. 1361-3.

Tkaczyk, E. R. (2017). Innovations and Developments in Dermatologic Non-invasive Optical Imaging and Potential Clinical Applications. Acta DermatoVenereologica. Suppl 218: 5-13.

Tomich, L. M., Pieper, J. B. \& Stern, A. W. (2017). Comparing dermoscopy and histological examination of normal equine skin. Veterinary Dermatology. 29(2):170-e63.

Wilkinson, G. T. \& Harvey, R. G. (1996). Atlas colorido de dermatologia dos pequenos animais. Guia para o diagnóstico. (2a ed.), Manole, 304p. 
Research, Society and Development, v. 10, n. 8, e28610817410, 2021

(CC BY 4.0) | ISSN 2525-3409 | DOI: http://dx.doi.org/10.33448/rsd-v10i8.17410

Zalaudek, I., Argenziano, G., Di Stefani, A. et al. (2006). Dermoscopy in general dermatology. Dermatology. 212: 7-18.

Zalaudek, I., Giacomel, J., Cabo, H., Di Stefani, A., Ferrara, G., Hofmann-Wellenhof, R. et al. (2008). Entodermoscopy: a new tool for diagnosing skin infections and infestations. Dermatology, v. 216, 14-23.

Zalaudek, I., Kreusch, J., Giacomel, J., Ferrara, G., Catricalà, C. \& Argenziano, G. (2010). How to diagnose nonpigmented skin tumors: a review of vascular structures seen with dermoscopy: part II. Nonmelanocytic skin tumors. Journal of the American Academy of Dermatology. 63: 377-86, 387-8.

Zanna, G., Auriemma, E., Arrighi, S. et al. (2015). Dermoscopic evaluation of skin in healthy cats. Veterinary Dermatology. 26: 14-17, e3-e4.

Zanna, G., Roccabianca, P., Zini, E., Legnani, S., Scarampella, F., Arrighi, S. \& Tosti, A. (2016). The usefulness of dermoscopy in canine pattern alopecia: a descriptive study. Veterinary Dermatology. Feb, 28 (1): 161-e34.

Zniszczynska, A. P., Szczepanik, M. \& Kalisz, G. (2021). Pilot videodermoscopic examination of hair and skin in arabian mare horeses during the winter season. Journal of Equine Veterinary Science. 99 (Suppl. 1). 